\title{
Isoelectric Focusing of Serum Apolipoprotein C-III as a Sensitive Screening Method for the Detection of O-glycosylation Disturbances
}

\author{
Nina Ondrušková', Tomáš Honzík', Jitka Kytnarová1, \\ Martin Matoulek ${ }^{2}$, Jiří Zeman ${ }^{1}$, Hana Hansíková ${ }^{1}$ \\ ${ }^{1}$ Department of Pediatrics and Adolescent Medicine, First Faculty of Medicine, \\ Charles University in Prague and General University Hospital in Prague, Prague, \\ Czech Republic; \\ ${ }^{2} 3^{\text {rd }}$ Department of Medicine - Department of Endocrinology and Metabolism, \\ First Faculty of Medicine, Charles University in Prague and General University \\ Hospital in Prague, Prague, Czech Republic
}

Received January 23, 2015; Accepted June 1, 2015.

Key words: Apolipoprotein C-III - O-glycosylation - Screening - Inherited disorders - Prader-Willi

\begin{abstract}
Apolipoprotein C-III (ApoC-III) is a glycoprotein carrying the most common O-linked glycan structure and is abundantly present in serum, what renders it a suitable marker for analysis of O-glycosylation abnormalities. Isoelectric focusing followed by a Western blot of ApoC-III, using PhastSystem ${ }^{\mathrm{TM}}$ Electrophoresis System (GE Healthcare), was introduced as a rather simple and rapid method for screening of certain subtypes of inherited glycosylation disorders. The study's aim was to establish this method in our laboratory, what included performing the analysis in a group of $\mathbf{1 7 0}$ healthy individuals to set the reference range of detected relative amounts of sialylated ApoC-III isoforms and to evaluate the gender- and age-dependent differences. A significant relative increase of asialoApoC-III with growing age was found. Secondly, we examined serum from patients with selected metabolic disorders and detected minor O-glycosylation changes
\end{abstract}

This study was supported by the grants GAUK 638512 (Charles University in Prague), IGA MZ NT/12166-5/2011, ExAM-CZ.1.05./2.1.00/03.0124 and RVO-VFN64165.

Mailing Address: RNDr. Hana Hansíková, PhD., Department of Pediatrics and Adolescent Medicine, First Faculty of Medicine, Charles University in Prague and General University Hospital in Prague, Ke Karlovu 2, 12808 Prague 2, Czech Republic; Phone: +420 224967 748; e-mail: hana.hansikova@If1.cuni.cz 
in diseases such as Prader-Willi syndrome, PGM1 (phosphoglucomutase 1) or MAN1B (class 1B alpha-1,2-mannosidase) deficiency. Our results show that this method allows for a sensitive detection of ApoC-III O-glycosylation status, however this might be modulated by several factors (i.e. nutrition, medication) whose exact role remains to be determined.

\section{Introduction}

Glycosylation is a functionally important and common posttranslational modification, with more than half of all human proteins estimated to be glycosylated (Christiansen et al., 2014). Congenital disorders of glycosylation (CDG) comprise a rapidly expanding family of metabolic diseases resulting from defects in glycosylation-related genes. Depending on which glycoprotein biosynthesis pathway is affected, more than 100 of $\mathrm{N}$ - and/or O-glycosylation disorders can be distinguished with a broad spectrum of clinical features (Freeze et al., 2014). In the more common group of $\mathrm{N}$-glycosylation disorders, psychomotor retardation is often present along with hypotonia, cerebellar hypoplasia, dysmorphy, strabismus, atypical fat distribution and coagulopathy; however, various other manifestations such as recurrent infections, gastrointestinal symptoms or deafness may occur. Combined $\mathrm{N}$ - and $\mathrm{O}$-glycosylation defects have a similar multi-systemic clinical picture, frequently including skeletal and skin anomalies. In contrast, isolated O-glycosylation disorders usually have a distinct phenotype defined by pronounced involvement of a single organ (brain, muscle) due to tissue-specific character of the enzymes involved in O-glycosylation (Wopereis et al., 2006b). As clinical report and basic laboratory tests are not sufficient for diagnosing CDG, selective screening methods are required to detect and categorize the glycosylation defect. Accordingly, additional experiments are performed (enzyme measurements, glycan structure analysis, immunocytochemical studies, etc.), before diagnosis is confirmed.

For CDG screening, serum $\mathrm{N}$-glycoprotein transferrin (TRF) and O-glycoprotein apolipoprotein C-III (ApoC-III) are used as markers. ApoC-III is a core 1 mucin type plasma protein synthesized predominantly in liver, acting as an inhibitor

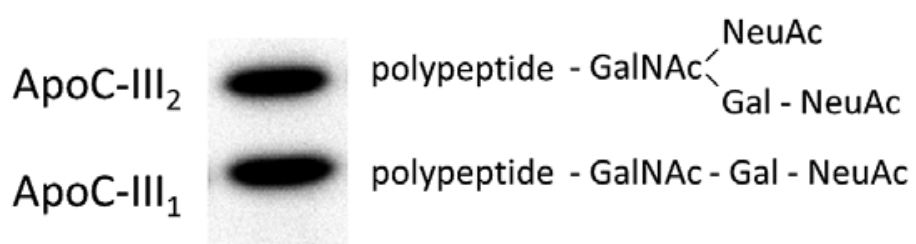

ApoC-III $\quad$ polypeptide - GalNAc-Gal

Figure 1 - Structure of ApoC-III O-glycosylated isoforms. Sialylated ApoC-III isoforms separated by isoelectric focusing (left) and the schematic structure of their corresponding glycan chains (right). 
of lipoprotein and hepatic lipases, i.e.VLDL or LDL clearance. It contains one branched oligosaccharide chain (glycan) attached via a hydroxy group to its polypeptide chain (threonine), and three isoforms with different sialic acid content can be detected:ApoC-III, ApoC-III ${ }_{1}$ and ApoC-III ${ }_{0}$ (di-, mono- and asialoform, respectively; Figure 1). In CDG types with impaired mucin O-glycosylation, which occurs exclusively in Golgi as opposed to $\mathrm{N}$-glycosylation localized both in ER (glycan assembly) and Golgi (further glycan modification), a pathological ApoC-III profile is observed characterized by decreased $\mathrm{ApoC}-\mathrm{III}_{2}$ and increased ApoC-III or ApoC-III ${ }_{0}$ (relative to total ApoC-III). To this date, aberrant ApoC-III sialylation has been described in glycosylation disorders including the deficiency of SLC35A1 (Wopereis et al., 2007), Conserved Oligomeric Golgi (COG) complex subunits 1, 2, 5, 6, 7 and 8 (Spaapen et al., 2005; Foulquier et al., 2006, 2007; Paesold-Burda et al., 2009; Lübbehusen et al., 2010; Kodera et al., 2015), ATP6V0A2 (Hucthagowder et al., 2009), ATP6V1A (Gardeitchik et al., 2014) and TMEM165 (Zeevaert et al., 2013). Recently, a new type of CDG due to phosphoglucomutase 1 (PGM1) deficiency was identified with an unusual TRF pattern in the CDG screening test, not clearly distinguishing which part of the glycosylation pathway is affected. No hypoglycosylation of ApoC-III was detected in the PGM1-CDG patient described by Pérez et al. (2013). Defect in the class 1B alpha-1,2-mannosidase (MAN1B1) gene manifests with a so called type 2 TRF pattern suggesting a disturbance in Golgi glycan modification, however no changes in ApoC-III glycosylation were reported (Van Scherpenzeel et al., 2014). The exact role of MAN1B1, originally thought of as an ER-resident mannosidase, has just lately been re-evaluated and it is currently presumed that the protein participates in Golgi-based protein biosynthesis quality control (Pan et al., 2013). Aside from the inborn defects in the glycosylation machinery, there are some pathophysiological factors that can influence the extent of serum ApoC-III sialylation. Documented secondary causes for ApoC-III hypoglycosylation include the acute phase of hemolytic uremic syndrome (HUS), where an enzyme cleaving sialic acid residues (neuraminidase) is released to circulation by Streptococcus pneumoniae, and Hutchinson Gilford progeria syndrome (HGSP) due to mutation in LMNA gene, possibly altering the biosynthesis of CMPNeuAc (Wopereis et al., 2007). Other examples are metabolic syndrome (Savinova et al., 2014) and Prader-Willi syndrome (Munce et al., 2010). On the other hand, excess ApoC-III sialylation resulting from CMP-NeuAc overproduction was demonstrated in sialuria, which is caused by defective GNE gene (Wopereis et al., 2006a). Increased ApoC-III ${ }_{2}$ to ApoC-III 1 ratio was also detected in individuals with chronic renal dysfunction (Holdsworth et al., 1982).

The study's aim was to determine the distribution of ApoC-III sialylated isoforms separated by isoelectric focusing (IEF) in sera from healthy individuals (a reference range) and to evaluate the gender- and age-dependent differences. The second aim was then to use the determined reference range for analysis of potential O-glycosylation abnormalities in patients with selected metabolic disorders. 


\section{Material and Methods}

\section{Material}

The control group consisted of 170 healthy individuals (age interval 1 day-42 years), 89 males and 81 females. To examine O-glycosylation abnormalities possibly arising secondarily to non-CDG etiologies, we analyzed 10 patients with Prader-Willi syndrome (s.), two patients with Rett s. and one individual each from Silver-Russell s., DiGeorge s., Gapo s., Schnitzler s., Marfan s., Stickler s., dyschondrosteosis and unexplained chronic renal dysfunction. The second tested group included 5 selected deficiencies of glycosylation enzymes or proteins (CDG) not directly involved in mucin O-glycosylation due to mutations in PMM2

Table 1 - Overview of selected clinical and laboratory parameters in the analyzed group of patients

\begin{tabular}{|c|c|c|c|c|c|c|}
\hline & Gender & Age & BMI & $\begin{array}{c}\text { Sleep } \\
\text { apnoea }\end{array}$ & $\begin{array}{l}\text { Triacyl- } \\
\text { glycerols } \\
\text { in serum } \\
\text { ( } \mathrm{mmol} / \mathrm{l} \text { ) }\end{array}$ & $\begin{array}{c}\text { Met- } \\
\text { formin } \\
\text { therapy }\end{array}$ \\
\hline PWS, Patient 1 & $\mathrm{~F}$ & $23 y$ & 49.7 & $Y$ & 3.50 & $\mathrm{~N}$ \\
\hline PWS, Patient 2 & $\mathrm{~F}$ & $27 y$ & 59.1 & Y & 1.07 & $Y$ \\
\hline PWS, Patient 3 & $\mathrm{~F}$ & $20 y$ & 45.8 & $\mathrm{~N}$ & 1.69 & $\mathrm{~N}$ \\
\hline PWS, Patient 4 & $M$ & $21 y$ & 43.4 & $\mathrm{~N}$ & 1.07 & $Y$ \\
\hline PWS, Patient 5 & $\mathrm{~F}$ & $33 y$ & 38.1 & $\mathrm{~N}$ & 1.05 & Y \\
\hline PWS, Patient 6 & $M$ & $17 y$ & 41.3 & $\mathrm{~N}$ & 0.62 & $Y$ \\
\hline PWS, Patient 7 & $\mathrm{~F}$ & $21 y$ & 51.9 & $\mathrm{~N}$ & 1.40 & $\mathrm{~N}$ \\
\hline PWS, Patient 8 & $\mathrm{~F}$ & $18 y$ & 27.0 & $\mathrm{~N}$ & 1.02 & $\mathrm{~N}$ \\
\hline PWS, Patient 9 & $M$ & $27 y$ & 56.1 & Y & 1.32 & $\mathrm{~N}$ \\
\hline PWS, Patient 10 & $M$ & $5 \mathrm{~m}$ & 14.5 & $\mathrm{~N}$ & NA & $\mathrm{N}$ \\
\hline Chronic renal dysfunction & $M$ & $2 y$ & 12.7 & $\mathrm{~N}$ & NA & $\mathrm{N}$ \\
\hline Rett syndrome, Patient 1 & $\mathrm{~F}$ & $2 y$ & 13.7 & $\mathrm{~N}$ & 1.10 & $\mathrm{~N}$ \\
\hline Rett syndrome, Patient 2 & $M$ & $7 y$ & 12.5 & $\mathrm{~N}$ & 2.22 & $\mathrm{~N}$ \\
\hline Silver-Russell syndrome & $M$ & $18 y$ & 12.8 & $\mathrm{~N}$ & 1.05 & $\mathrm{~N}$ \\
\hline DiGeorge syndrome & $\mathrm{F}$ & $12 y$ & 16.5 & $\mathrm{~N}$ & 0.46 & $\mathrm{~N}$ \\
\hline Gapo syndrome & $M$ & $18 y$ & NA & NA & NA & NA \\
\hline Schnitzler syndrome & $M$ & $46 y$ & NA & NA & NA & NA \\
\hline Marfan syndrome & $M$ & $27 y$ & NA & $N$ & NA & $N$ \\
\hline Stickler syndrome & $\mathrm{F}$ & $32 y$ & 23.1 & $\mathrm{~N}$ & 0.80 & $\mathrm{~N}$ \\
\hline Dyschondrosteosis & $\mathrm{F}$ & $32 y$ & 32.5 & $\mathrm{~N}$ & 0.77 & $\mathrm{~N}$ \\
\hline PMM2-CDG & $\mathrm{F}$ & $24 y$ & 23.7 & $\mathrm{~N}$ & 1.20 & $\mathrm{~N}$ \\
\hline EXT1-CDG & $\mathrm{F}$ & $3 y$ & NA & $\mathrm{N}$ & NA & $\mathrm{N}$ \\
\hline NGBR deficiency & $M$ & $11 \mathrm{~m}$ & 14.7 & $\mathrm{~N}$ & NA & $\mathrm{N}$ \\
\hline PGM1-CDG & $M$ & $7 y$ & 21.7 & $\mathrm{~N}$ & 0.47 & $\mathrm{~N}$ \\
\hline MAN1B1-CDG & $M$ & $10 y$ & NA & NA & NA & NA \\
\hline
\end{tabular}

PWS - Prader-Willi syndrome; F - female; $M$ - male; $y$ - years; $m$ - months; BMI - body mass index; NA - not analyzed/no information 
(phosphomannomutase 2), PGM1, EXT1 (exostosin glycosyltransferase 1), MAN1B1 and NGBR (Nogo-B receptor) genes, one patient from each group. Selected clinical and laboratory data of the analyzed patients are shown in Table 1 . Serum was separated by centrifugation $(630 \mathrm{~g}, 10 \mathrm{~min})$ and stored at $-20^{\circ} \mathrm{C}$ until tested. Analyses were performed with informed consent from each participant or their parents.

Isoelectric focusing (IEF) and Western blot of ApoC-III

IEF of ApoC-III was carried out as described by Wopereis et al. (2003), with minor modifications. Dry IEF gels (17-0677-01, GE Healthcare) were incubated in a rehydrating solution (8.3 M urea, 4\% (V/V) Pharmalyte $\mathrm{pH}$ 4.2-4.9 (17-0562-01, GE Healthcare), 2\% (V/V) Ampholine pH 3.5-5 (80-1125-89, GE Healthcare)) for $1.5 \mathrm{~h}$. Serum was centrifuged at $6600 \mathrm{~g}$ for $2 \mathrm{~min}$ and the clear middle fraction (non-diluted) was used as a sample. The separation of samples (approx. $0.5 \mu \mathrm{l}$ each) by isoelectric focusing was performed on PhastSystem ${ }^{\mathrm{TM}}$ Electrophoresis System (GE Healthcare). After the run was completed, gels were briefly washed in transfer buffer ( $25 \mathrm{mM}$ Tris, $192 \mathrm{mM}$ glycine, 20\% methanol) and proteins were blotted onto nitrocellulose membranes (pre-equilibrated in transfer buffer) by diffusion at $60{ }^{\circ} \mathrm{C}$ for $1 \mathrm{~h}$. The membranes were then blocked in milk solution $(5 \%(\mathrm{w} / \mathrm{V})$ in PBST: PBS with $0.5 \%(\mathrm{~V} / \mathrm{V})$ Tween 20), labelled with primary ApoC-III antibody (06501707, Biotrend) diluted 1:1000 in BSA solution ( $2 \%(w / V)$ in PBST) and anti-rabbit secondary antibody conjugated to horseradish peroxidase (A-0545, Sigma; 1:5000 in $2 \%(w / V)$ milk in PBST). Each incubation was carried out for $1 \mathrm{~h}$, with $3 \times 10 \mathrm{~min}$ washes (PBS with $0.05 \%(\mathrm{~V} / \mathrm{V})$ Tween 20 ) in between. After the final washing in PBS $(2 \times 5 \mathrm{~min})$, blots were visualized by chemiluminiscence (SuperSignal West Femto Substrate, Thermo Scientific) using GBOX Imaging System (Syngene). The profiles of ApoC-III isoforms were quantified densitometrically with Quantity One software (Bio-Rad). Examples of both normal and hypoglycosylated ApoC-III profiles are shown in Figure 2. The coefficient of variation for intra-run variability $\left(\mathrm{CV}_{1}\right)$ was determined by evaluating 8 profiles of serum from one healthy control analyzed

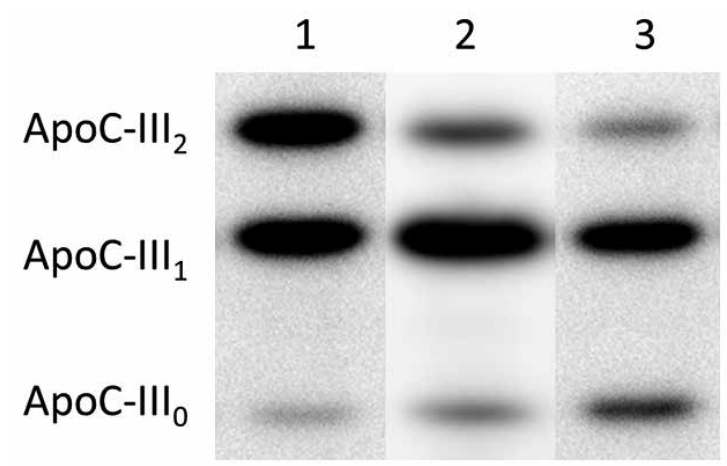

Figure 2 - Comparison of normal vs. hypoglycosylated ApoC-III IEF profiles. The picture shows an example of a physiological ApoC-III profile (1) and pathological profiles characterized by a relative decrease of ApoC-III ${ }_{2}$ with relatively increased ApoC-III 1 (2) or ApoC-III 0 (3). The ratios vary in different syndromes and patients. 
within one gel/run, and the same control sample was analyzed in 35 different gels/runs for the calculation of the coefficient of variation for inter-run variability $\left(\mathrm{CV}_{2}\right) . \mathrm{CV}_{1}: 8.4,6.8,22.1 ; \mathrm{CV}_{2}: 6.7,4.9,23.5$ (for ApoC-III, ApoC-III ${ }_{1}$ and ApoC-III $\mathrm{O}_{0}$, respectively).

\section{Statistical analysis}

Because of the non-normal distribution of the data, the reference range was calculated using $2.5 \%$ and $97.5 \%$ sample quantiles. Gender-determined variations in given age categories were tested with non-parametric Wilcoxon test. Differences between the age categories were tested for both genders using Kruskal-Wallis test. A statistically significant difference was set as $p<0.05$.

\section{Results}

Individuals from the analyzed control group were divided into three separate categories based on their age: 1) less than 2 years old, 2) 2-6 years old, and 3) 7-42 years old. The statistically evaluated distribution of ApoC-III sialylated isoforms - including the median, mean values and reference range - determined for the whole group is shown in Figure 3.

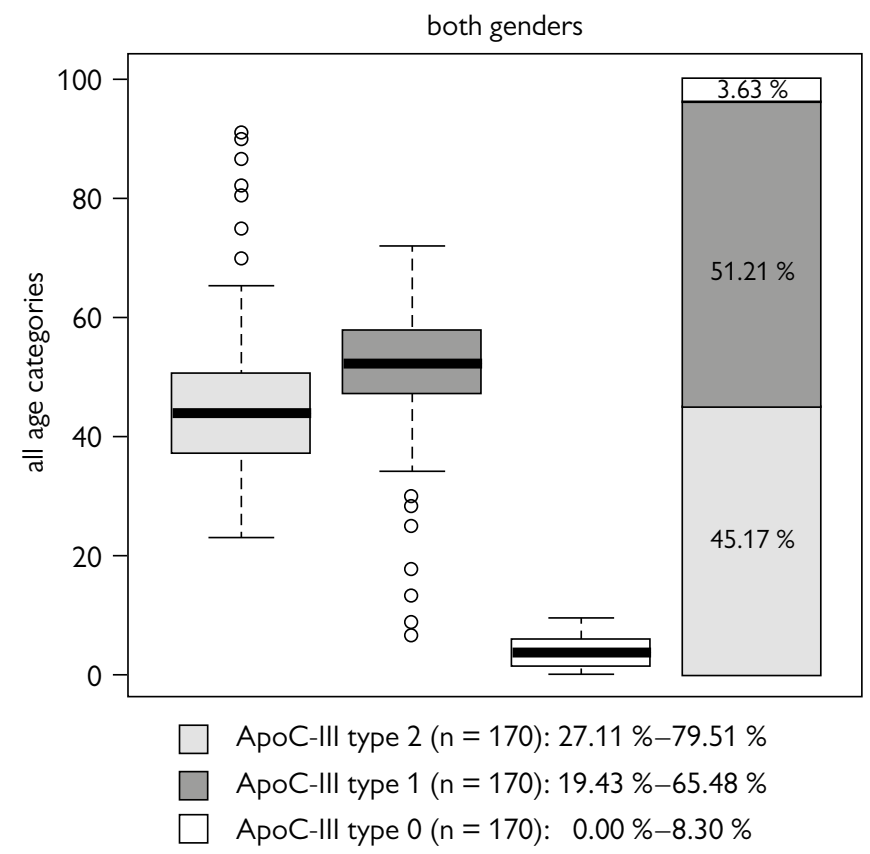

Figure 3 - Distribution of ApoC-III sialylated isoforms separated by isoelectric focusing of serum samples, determined in a group of healthy individuals $(n=170)$ of both genders in the age interval 1 day-42 years. The bold line inside box-plot represents median, the column graph on the right shows mean values. Intervals below are the reference range. 


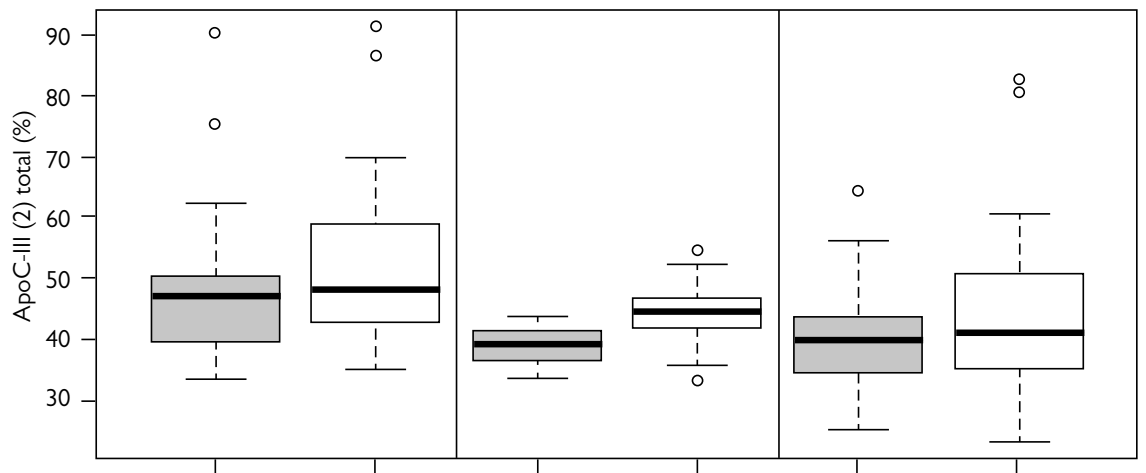

female, $<2$ y male, $<2$ y female, 2-6y male, $2-6$ y female, $7-42$ y male, $7-42$ y
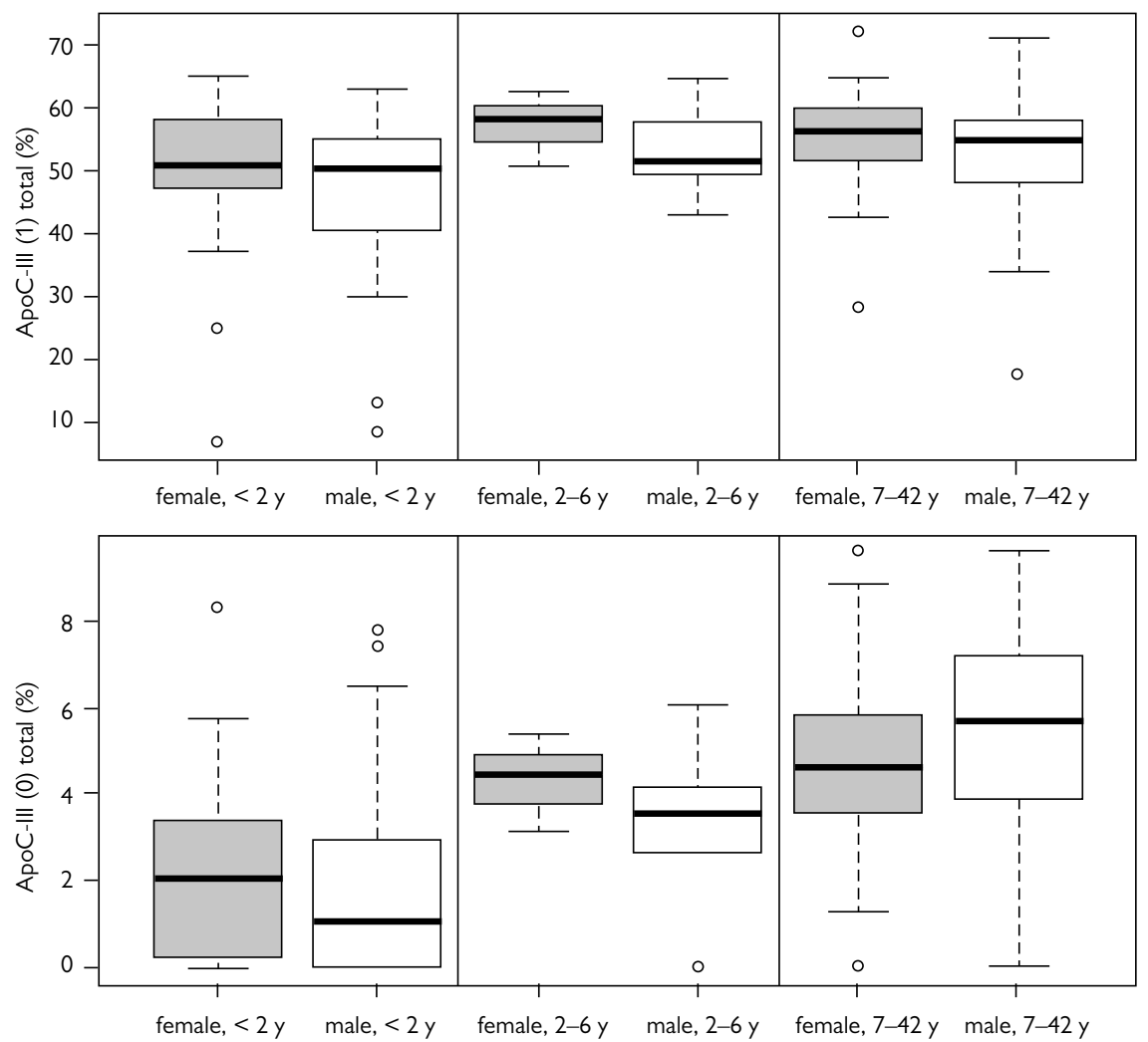

Figure 4 - Evaluation of gender- and age-dependent differences in the distribution of ApoC-III sialylated isoforms separated by isoelectric focusing of serum samples, determined in a group of healthy individuals $(n=170)$ of both genders (89 males, 81 females) in three different age categories: 1) < 2 y $(n=70), 2) 2-6$ y $(n=16)$ and 3) 7-42 y $(n=84)(y-$ years). The bold line inside box-plots represents median. 1) Wilcoxon test results (female vs. male) for a) ApoC-III $: p=0.238(<2 y) ; 0.157(2-6 y) ; 0.345(7-42 y)$, b) ApoC- $I I I_{1}: p=0.226(<2 y) ; 0.281$ $(2-6$ y); 0.181 (7-42 y) and c) ApoC-III $: p=0.522(<2$ y); 0.380 (2-6 y); 0.165 (7-42 y). 2) Kruskal-Wallis test results (age dependent differences) for a) ApoC-III $I_{2}: p=0.002$ (female $\left.-F\right) ; 0.008$ (male $-M$ ), b) ApoC-III $1_{1}$ : $p=0.041(F) ; 0.110(M)$ and c) ApoC-III $I_{0} p<0.001$ (F); $<0.001(M)$. 
No statistically significant changes in ApoC-III sialylated isoforms' relative amounts were found between males and females ( $p>0.05$, Figure 4). However, regarding age-dependent differences, we noted a relative increase of ApoC-III with growing age, accompanied by a relative decrease of the ApoC-III 2 isoform. This trend was calculated as statistically significant, for both genders (the lowest value $\mathrm{p}<0.001$ was obtained when comparing ApoC-III 0 content between groups $<2$ years and $7-42$ years; Figure 4 ).

Pathological ApoC-III profile was defined as a combination of relatively decreased ApoC-III 2 and relatively increased ApoC-III ${ }_{1}$ or ApoC-III compared to controls. Out of the tested non-CDG patients, all showed physiological ApoC-III profile except for three individuals with Prader-Willi s. (PWS; Patients 1, 3 and 7 in Table 1) and a boy with chronic renal dysfunction. As expected, normal O-glycosylation of ApoC-III was found in patients with genetic defects in PMM2, EXT1 and NGBR. Multiple serum samples were analyzed from a boy with PGM1 deficiency, displaying from borderline to pathological pattern, and a slight ApoC-III hypoglycosylation was also seen in patient with MAN1B1 defect. The abnormal ApoC-III profiles are depicted in Figure 5 and the quantification of all analyzed ApoC-III profiles is shown in Table 2.

\section{Discussion}

To establish a screening assay for detecting O-glycosylation abnormalities in serum, we performed separation of ApoC-III sialylated isoforms by isoelectric focusing, followed by a Western blot detection - a method developed by Wopereis et al. (2003) - and determined a reference range of their relative amounts in our control group of 170 healthy subjects. While we found no variations between genders, there was a statistically significant relative decrease of ApoC-III 2 and increase of ApoC-III 0 with growing age. It underlines the need to consider the patient's age when evaluating their profile. This finding was in agreement with previous

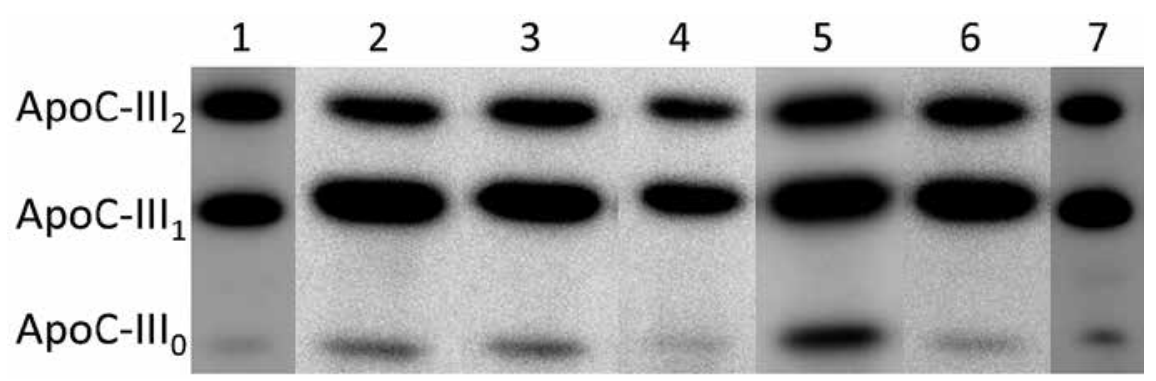

Figure 5 -ApoC-III hypoglycosylation detected in the analyzed group of patients. Serum samples were separated by isoelectric focusing, followed by a Western blot detection of ApoC-III. Relatively decreased ApoC-III ${ }_{2}$ and increased ApoC-III ${ }_{1}$ and/or ApoC-III ${ }_{0}$ compared to a control (lane 1) were seen in three patients with Prader-Willi syndrome (2-4), unexplained chronic renal dysfunction (5), PGM1 (6) and MAN1B1 (7) deficiency. 


\section{Table 2 - Relative amounts of sialylated ApoC-III isoforms in the analyzed group of patients}

\begin{tabular}{|c|c|c|c|c|}
\hline & \multirow{2}{*}{ Age } & \multicolumn{3}{|c|}{ Percentage of total ApoC-III (\%) } \\
\hline & & Disialo-(2) & Monosialo-(1) & Asialo-(0) \\
\hline Reference range & $1 d-42 y$ & $27.1-79.5$ & $19.4-65.5$ & $0-8.3$ \\
\hline $\begin{array}{l}\text { PWS, Patient } 1 \\
\text { (lane } 2 \text { in Figure 5) }\end{array}$ & $23 y$ & 24.2 & 73.3 & 2.5 \\
\hline PWS, Patient 2 & $27 y$ & 39.9 & 51.1 & 9.0 \\
\hline PWS, Patient 3 (lane 3) & $20 y$ & 27.3 & 70.3 & 2.4 \\
\hline PWS, Patient 4 & $21 y$ & 37.2 & 57.4 & 5.4 \\
\hline PWS, Patient 5 & $33 y$ & 48.4 & 48.8 & 2.9 \\
\hline PWS, Patient 6 & $17 y$ & 42.6 & 57.5 & ND \\
\hline PWS, Patient 7 (lane 4) & $21 y$ & 28.2 & 71.9 & ND \\
\hline PWS, Patient 8 & $18 y$ & 39.2 & 58.8 & 2.0 \\
\hline PWS, Patient 9 & $27 y$ & 35.7 & 61.3 & 3.1 \\
\hline PWS, Patient 10 & $5 \mathrm{~m}$ & 46.8 & 51.3 & 1.9 \\
\hline $\begin{array}{l}\text { Chronic renal dysfunction } \\
\text { (lane 5) }\end{array}$ & $2 y$ & 25.0 & 62.3 & 12.7 \\
\hline Rett syndrome, Patient 1 & $2 y$ & 40.8 & 55.2 & 4.0 \\
\hline Rett syndrome, Patient 2 & $7 y$ & 34.1 & 59.4 & 6.5 \\
\hline Silver-Russell syndrome & $18 y$ & 50.8 & 47.4 & 1.8 \\
\hline DiGeorge syndrome & $12 y$ & 46.6 & 51.9 & 1.6 \\
\hline Gapo syndrome & $18 y$ & 43.8 & 51.1 & 5.2 \\
\hline Schnitzler syndrome & $46 y$ & 39.9 & 57.2 & 2.9 \\
\hline Marfan syndrome & $27 y$ & 55.9 & 37.7 & 6.4 \\
\hline Stickler syndrome & $32 y$ & 37.2 & 60.0 & 2.8 \\
\hline Dyschondrosteosis & $32 y$ & 39.0 & 60.0 & 1.0 \\
\hline PMM2-CDG & $24 y$ & 34.8 & 59.8 & 5.4 \\
\hline EXT1-CDG & $3 y$ & 59.0 & 39.2 & 1.9 \\
\hline NGBR deficiency & $11 \mathrm{~m}$ & 43.1 & 54.4 & 2.5 \\
\hline PGM1-CDG (lane 6) & $7 y$ & 27.4 & 71.7 & 1.0 \\
\hline MAN1B1-CDG (lane 7) & $10 y$ & 27.9 & 69.3 & 2.8 \\
\hline
\end{tabular}

PWS - Prader-Willi syndrome; $d$ - day; $y$ - years; $m$ - months; ND - not detected

report by Wopereis et al. (2007), who, in addition, found higher relative levels of ApoC-III ${ }_{2}$ in premature neonates than in full-term newborns. The observed agedependent changes of ApoC-III sialylation are, in our opinion, likely downstream consequences of alterations in lipid metabolism associated with development/aging. It is unknown whether they directly hold any implications for the individual's health status, however in a study done by Tertov et al. (1996), it was determined that the circulating immune complexes containing low density lipoprotein (CIC-LDL), present in the blood of patients with coronary atherosclerosis, have considerably (2.1-fold) lower sialic acid content than native LDL.

It has been long known that increased ApoC-III level is associated with elevated triacylglycerols (TAG) in plasma (Kashyap et al., 1981), and is considered a valuable 
marker for the risk of coronary heart disease (Sacks et al., 2000). Overexpressing ApoC-III in transgenic mice elevated TAG levels (Ito et al., 1990), while disruption of ApoC-III gene caused hypotriglyceridemia (Maeda et al., 1994). Vice versa, high total plasma TAG and VLDL-TAG in hypertriglyceridemia lead to accumulation of ApoC-III in triglyceride rich lipoproteins (TRL), resulting from both increased transfer of ApoC-III to TRL and increased hepatic production of VLDL ApoC-III (Marcoux et al., 2001). Recently, Savinova et al. (2014) found an increase of total ApoC-III (approx. 1.4-fold) together with its slightly reduced glycosylation in 56 subjects with metabolic syndrome, compared to controls. In their study, VLDL fraction of the patients contained $33 \%$ of ApoC-III, $58 \%$ of ApoC-III ${ }_{1}$ and $9 \%$ of ApoC-III , whereas the controls had $40 \%, 52 \%$ and $8 \%$, respectively. They point out that ApoC-III sialylation is responsive to prescription of metformin, as well as of omega- 3 fatty acids and niacin (author's note: these medications are known to lower plasma TAG). Interestingly, some experiments in rats also documented alterations in the relative amounts of ApoC-III sialylated isoforms under different dietary conditions, where carbohydrate-rich diet, accompanied by a rise in plasma TAG levels, induced an increase of plasma ApoC-III (Holdsworth et al., 1982). All the data above suggest a direct relationship between TAG levels and ApoC-III hyposialylation, however the underlying mechanism is currently not clear. At this point we can only speculate whether this is caused by impaired ApoC-III catabolism and consequent rises in relative amounts of its hyposialylated isoforms, or perhaps due to the increased production rate of new ApoC-III molecules and their secretion through Golgi apparatus (where sialylation occurs), the glycosylation machinery is overwhelmed and fails to fully modify all the passing glycoproteins. We confirm an observation of pathological ApoC-III profiles with decreased ApoC-III ${ }_{2}$ and increased ApoC- $-I I_{1}(>70 \%)$ in 3 individuals (positive) and a borderline profile with increased ApoC-III $(9 \%)$ in one individual (Patient 2 in Table 1), out of 10 analyzed patients with confirmed PWS. While Munce et al. (2010) found a correlation between abnormal ApoC-III profiles in PWS patients and sleep abnormalities, in our study only one positive patient suffered from a sleep apnoea. They reasoned that the observed glycosylation abnormality could be caused by deficiency in regulating protein degradation, based on their finding of two genes with putative roles as E3 ubiquitin ligases in the common deleted region. No relationship between aberrant ApoC-III and BMI was detected by Munce et al. (2010) or us, however we noted higher TAG ( $\geq 1.4 \mathrm{mmol} / \mathrm{l})$ in all positive PWS patients compared to the others, supporting our assumption that ApoC-III hyposialylation in PWS is attributable to increased plasma TAG levels. We believe that drug treatments could have affected the observed results in our study, i.e. normalized the possible hyposialylation of ApoC-III through lowering plasma TAG.

Ooi et al. (2011) studied ApoC-III metabolism in subjects with moderate chronic kidney disease and found that they had significantly higher plasma ApoC-III concentrations, and the selected isoform for analysis, ApoC-III, had 
significantly lower fractional catabolic rate. They hypothesize that elevated ApoC-III sialylation documented by Holdsworth et al. (1982) could render ApoC-III-containing TRL particles less suitable for lipolytic degradation. Moreover, the delayed ApoC-III catabolism could be due to impaired renal function as kidney is partly involved in the removal of ApoC-III from plasma. This contradicts our finding of a hypoglycosylated ApoC-III pattern ( $\downarrow$ ApoC-III,$\uparrow$ ApoC-III $)$ in a patient with chronic renal dysfunction as the predominant symptom. We cannot exclude the potential effect of elevated TAG level as it was not measured in the analyzed serum. Interestingly, hypoglycosylation linked to renal dysfunction is well documented in IgA nephropathy (IgAN), characterized by specific undergalactosylation of IgA1 O-glycans (Narita and Gejyo, 2008). They suggest that there is no primary abnormality in O-galactosylation pathway in $B$ lymphocytes, but the IgA1 glycosylation defect is rather secondary to aberrant immunological reaction with IgA1 overproduction. In addition, recent studies have found decreased expression of GALNT2 (the corresponding enzyme polypeptide $\mathrm{N}$-acetylgalactosaminyltransferase initiates mucin type O-glycosylation) modulated by miRNA in IgA1-producing cells of IgAN patients (Serino et al., 2015). The finding is intriguing, since decreased GALNT2 expression was also detected in circulating blood cells of obese diabetic patients, probably triggered by their hyperglycemia (Marucci et al., 2013). Moreover, certain SNPs in GALNT2 gene were found to be associated with plasma TAG and cholesterol levels (Kathiresan et al., 2008). Thus, altered GALNT2 activity might be linked to various conditions of metabolic derangement. While our patient did not present with features of IgAN, the observed ApoC-III hypoglycosylation could be secondary to yet unrecognized metabolic factor associated with his severe phenotype.

PGM1 deficiency indirectly lowers the intracellular concentration of UDP-Gal (Tegtmeyer et al., 2014), one of the substrates needed for mucin type $1 \mathrm{O}$-glycosylation, what could possibly explain our finding of reduced ApoC-III sialylation in the analyzed patient. However, the extent of detected hypoglycosylation was variable in serum samples withdrawn at different periods and sometimes the abnormal changes were rather subtle. Xia et al. (2013) performed LC/MS-MS analysis to quantify selected plasma O-glycan structures in various CDG subtypes and, interestingly, their PGM1-CDG patient had increased T/ST-antigen ratio (Gal-GalNAc/Sia-Gal-GalNAc) similar to those reported in galactosemia. The other analyzed PGM1-CDG patient who was on galactose treatment showed normal pattern, supporting the idea that the observed glycosylation disturbances in PGM1-CDG result from reduced UDP-Gal pool, which was also reported in galactosemic patients ( $\mathrm{Ng}$ et al., 1989). Borderline ApoC-III hyposialylation was detected in our patient with pathogenic mutation in MAN1B1 gene. Its deficiency disturbs Golgi morphology and impairs Golgi anterograde trafficking as studied in MAN1B1-depleted cells (Péanne et al., 2014), likely affecting both $\mathrm{N}$ - and O-glycosylation. 


\section{Conclusion}

We determined a reference range and assessed gender- and age-dependent differences of the distribution of serum apolipoprotein C-III sialylated isoforms detected by Western blot after isoelectric focusing. Besides finding a mild ApoC-III hypoglycosylation in some individuals with Prader-Willi syndrome what had already been documented elsewhere, we suggest it is linked to plasma triacylglycerol levels. We also show, for the first time, slightly reduced O-glycosylation of ApoC-III in patients with PGM1 and MAN1B1 deficiency. Thus, we conclude the established method is sufficiently sensitive and suitable for screening of mucin type $1 \mathrm{O}$-glycosylation disturbances. Our results also demonstrate the importance of multiple analyses in the same individual. Further studies should be done to elucidate how patients' medication and diet might influence the outcome of the analysis.

Acknowledgements:The authors thank to Václav Caapek, PhD., for executing the statistical analyses.

\section{References}

Christiansen, M. N., Chik, J., Lee, L., Anugraham, M., Abrahams, J. L., Packer, N. H. (2014) Cell surface protein glycosylation in cancer. Proteomics 14(4-5), 525-546.

Foulquier, F., Vasile, E., Schollen, E., Callewaert, N., Raemaekers, T., Quelhas, D., Jaeken, J., Mills, P., Winchester, B., Krieger, M., Annaert, W., Matthijs, G. (2006) Conserved oligomeric Golgi complex subunit 1 deficiency reveals a previously uncharacterized congenital disorder of glycosylation type II. Proc. Natl. Acad. Sci. U. S. A. 103(10), 3764-3769.

Foulquier, F., Ungar, D., Reynders, E., Zeevaert, R., Mills, P., García-Silva, M. T., Briones, P., Winchester, B., Morelle,W., Krieger, M., Annaert, W., Matthijs, G. (2007) A new inborn error of glycosylation due to a Cog8 deficiency reveals a critical role for the Cog1-Cog8 interaction in COG complex formation. Hum. Mol. Genet. 16(7), 717-730.

Freeze, H. H., Chong, J. X., Bamshad, M. J., Ng, B. G. (2014) Solving glycosylation disorders: Fundamental approaches reveal complicated pathways. Am. J. Hum. Genet. 94(2), 161-175.

Gardeitchik, T., Mohamed, M., Korenke, C., Van Asbeck, E., Van Kraaij, S., Monique, V. S., Lefeber, D., Wevers, R., Morava, E. (2014) A novel genetic defect connecting cutis laxa to congenital disorders of glycosylation. J. Inherit. Metab. Dis. 37, 163 (Suppl. 1).

Holdsworth, G., Stocks, J., Dodson, P., Galton, D. J. (1982) An abnormal triglyceride-rich lipoprotein containing excess sialylated apolipoprotein C-III. J. Clin. Invest. 69(4), 932-939.

Hucthagowder, V., Morava, E., Kornak, U., Lefeber, D. J., Fischer, B., Dimopoulou, A., Aldinger, A., Choi, J., Davis, E. C., Abuelo, D. N., Adamowicz, M., Al-Aama, J., Basel-Vanagaite, L., Fernandez, B., Greally, M. T., GillessenKaesbach, G., Kayserili, H., Lemyre, E., Tekin, M., Türkmen, S., Tuysuz, B., Yüksel-Konuk, B., Mundlos, S., Van Maldergem, L., Wevers, R. A., Urban, Z. (2009) Loss-of-function mutations in ATP6V0A2 impair vesicular trafficking, tropoelastin secretion and cell survival. Hum. Mol. Genet. 18(12), 2149-2165.

Ito, Y., Azrolan, N., O’Connell, A., Walsh, A., Breslow, J. L. (1990) Hypertriglyceridemia as a result of human apo CIII gene expression in transgenic mice. Science 249(4970), 790-793.

Kashyap, M. L., Srivastava, L. S., Hynd, B. A., Gartside, P. S., Perisutti, G. (1981) Quantitation of human apolipoprotein C-III and its subspecie by radioimmunoassay and analytical isoelectric focusing: 
Abnormal plasma triglyceride-rich lipoprotein apolipoprotein C-III subspecie concentrations in hypertriglyceridemia. J. Lipid Res. 22(5), 800-810.

Kathiresan, S., Melander, O., Guiducci, C., Surti, A., Burtt, N. P., Rieder, M. J., Cooper, G. M., Roos, C., Voight, B. F., Havulinna, A. S., Wahlstrand, B., Hedner, T., Corella, D., Tai, E. S., Ordovas, J. M., Berglund, G., Vartiainen, E., Jousilahti, P., Hedblad, B., Taskinen, M. R., Newton-Cheh, C., Salomaa, V., Peltonen, L., Groop, L., Altshuler, D. M., Orho-Melander, M. (2008) Six new loci associated with blood low-density lipoprotein cholesterol, high-density lipoprotein cholesterol or triglycerides in humans. Nat. Genet. 40(2), 189-197.

Kodera, H., Ando, N., Yuasa, I., Wada, Y., Tsurusaki, Y., Nakashima, M., Miyake, N., Saitoh, S., Matsumoto, N., Saitsu, H. (2015) Mutations in COG2 encoding a subunit of the conserved oligomeric Golgi complex cause a congenital disorder of glycosylation. Clin. Genet. 87(5), 455-460.

Lübbehusen, J., Thiel, C., Rind, N., Ungar, D., Prinsen, B. H., De Koning, T. J., Van Hasselt, P. M., Körner, C. (2010) Fatal outcome due to deficiency of subunit 6 of the conserved oligomeric Golgi complex leading to a new type of congenital disorders of glycosylation. Hum. Mol. Genet. 19(18), 3623-3633.

Maeda, N., Li, H., Lee, D., Oliver, P., Quarfordt, S. H., Osada, J. (1994) Targeted disruption of the apolipoprotein C-III gene in mice results in hypotriglyceridemia and protection from postprandial hypertriglyceridemia. J. Biol. Chem. 269(38), 23610-23616.

Marcoux, C., Tremblay, M., Fredenrich, A., Davignon, J., Cohn, J. S. (2001) Lipoprotein distribution of apolipoprotein C-III and its relationship to the presence in plasma of triglyceride-rich remnant lipoproteins. Metabolism 50(1), 112-119.

Marucci, A., Di Mauro, L., Menzaghi, C., Prudente, S., Mangiacotti, D., Fini, G., Lotti, G., Trischitta, V., Di Paola, R. (2013) GALNT2 expression is reduced in patients with type 2 diabetes: Possible role of hyperglycemia. PLoS One 8(7), e70159.

Munce, T., Heussler, H. S., Bowling, F. G. (2010) Analysis of N- and O-linked protein glycosylation in children with Prader-Willi syndrome. J. Intellect. Disabil. Res. 54(10), 929-937.

Narita, I., Gejyo, F. (2008) Pathogenetic significance of aberrant glycosylation of IgA1 in IgA nephropathy. Clin. Exp. Nephrol. 12(5), 332-338.

Ng, W. G., Xu, Y. K., Kaufman, F. R., Donnell, G. N. (1989) Deficit of uridine diphosphate galactose in galactosaemia. J. Inherit. Metab. Dis. 12(3), 257-266.

Ooi, E. M., Chan, D. T., Watts, G. F., Chan, D. C., Ng, T.W., Dogra, G. K., Irish, A. B., Barrett, P. H. (2011) Plasma apolipoprotein C-III metabolism in patients with chronic kidney disease. J. Lipid Res. 52(4), 794-800.

Paesold-Burda, P., Maag, C., Troxler, H., Foulquier, F., Kleinert, P., Schnabel, S., Baumgartner, M., Hennet, T. (2009) Deficiency in COG5 causes a moderate form of congenital disorders of glycosylation. Hum. Mol. Genet. 18(22), 4350-4356.

Pan, S., Cheng, X., Sifers, R. N. (2013) Golgi-situated endoplasmic reticulum $\alpha-1,2$-mannosidase contributes to the retrieval of ERAD substrates through a direct interaction with $\gamma$-COP. Mol. Biol. Cell 24(8), 1111-1121.

Péanne, R., Rymen, D., Jurisch-Yaksi, N., Foulquier, F., Annaert, W., Matthijs, G. (2014) MAN1B1-CDG: How stressed-out can the Golgi be? Glycobiology 24(11), 1105.

Pérez, B., Medrano, C., Ecay, M. J., Ruiz-Sala, P., Martínez-Pardo, M., Ugarte, M., Pérez-Cerdá, C. (2013) A novel congenital disorder of glycosylation type without central nervous system involvement caused by mutations in the phosphoglucomutase 1 gene. J. Inherit. Metab. Dis. 36(3), 535-542.

Sacks, F. M., Alaupovic, P., Moye, L. A., Cole, T. G., Sussex, B., Stampfer, M. J., Pfeffer, M. A., Braunwald, E. (2000) VLDL, apolipoproteins $B, C I I I$, and $E$, and risk of recurrent coronary events in the cholesterol and recurrent events (CARE) trial. Circulation 102(16), 1886-1892.

Savinova, O. V., Fillaus, K., Jing, L., Harris, W. S., Shearer, G. C. (2014) Reduced apolipoprotein glycosylation in patients with the metabolic syndrome. PLoS One 9(8), e104833. 
Serino, G., Sallustio, F., Curci, C., Cox, S. N., Pesce, F., De Palma, G., Schena, F. P. (2015) Role of let-7b in the regulation of $\mathrm{N}$-acetylgalactosaminyltransferase 2 in IgA nephropathy. Nephrol. Dial. Transplant. (Epub ahead of print)

Spaapen, L. J., Bakker, J. A., van der Meer, S. B., Sijstermans, H. J., Steet, R. A., Wevers, R. A., Jaeken, J. (2005) Clinical and biochemical presentation of siblings with COG-7 deficiency, a lethal multiple O- and N-glycosylation disorder. J. Inherit. Metab. Dis. 28(5), 707-714.

Tegtmeyer, L. C., Rust, S., Van Scherpenzeel, M., Ng, B. G., Losfeld, M. E., Timal, S., Raymond, K., He, P., Ichikawa, M., Veltman, J., Huijben, K., Shin, Y. S., Sharma, V., Adamowicz, M., Lammens, M., Reunert, J., Witten, A., Schrapers, E., Matthijs, G., Jaeken, J., Rymen, D., Stojkovic, T., Laforêt, P., Petit, F., Aumaître, O., Czarnowska, E., Piraud, M., Podskarbi, T., Stanley, C.A., Matalon, R., Burda, P., Seyyedi, S., Debus, V., Socha, P., Sykut-Cegielska, J., Van Spronsen, F., De Meirleir, L., Vajro, P., DeClue, T., Ficicioglu, C., Wada, Y., Wevers, R. A., Vanderschaeghe, D., Callewaert, N., Fingerhut, R., Van Schaftingen, E., Freeze, H. H., Morava, E., Lefeber, D. J., Marquardt, T. (2014) Multiple phenotypes in phosphoglucomutase 1 deficiency. N. Engl. J. Med. 370(6), 533-542.

Tertov, V.V., Sobenin, I. A., Orekhov, A. N., Jaakkola, O., Solakivi, T., Nikkari, T. (1996) Characteristics of low density lipoprotein isolated from circulating immune complexes. Atherosclerosis 122(2), 191-199.

Van Scherpenzeel, M., Timal, S., Rymen, D., Hoischen, A., Wuhrer, M., Hipgrave-Ederveen, A., Grunewald, S., Peanne, R., Saada, A., Edvardson, S., Grønborg, S., Ruijter, G., Kattentidt-Mouravieva, A., Brum, J. M., Freckmann, M. L., Tomkins, S., Jalan, A., Prochazkova, D., Ondruskova, N., Hansikova, H., Willemsen, M. A., Hensbergen, P. J., Matthijs, G., Wevers, R. A., Veltman, J. A., Morava, E., Lefeber, D. J. (2014) Diagnostic serum glycosylation profile in patients with intellectual disability as a result of MAN1B1 deficiency. Brain 137(Pt 4), 1030-1038.

Wopereis, S., Grünewald, S., Morava, E., Penzien, J. M., Briones, P., García-Silva, M. T., Demacker, P. N., Huijben, K. M., Wevers, R. A. (2003) Apolipoprotein C-III isofocusing in the diagnosis of genetic defects in O-glycan biosynthesis. Clin. Chem. 49(11), 1839-1845.

Wopereis, S., Abd Hamid, U. M., Critchley, A., Royle, L., Dwek, R. A., Morava, E., Leroy, J. G., Wilcken, B., Lagerwerf, A. J., Huijben, K. M., Lefeber, D. J., Rudd, P. M., Wevers, R. A. (2006a) Abnormal glycosylation with hypersialylated O-glycans in patients with Sialuria. Biochim. Biophys. Acta 1762(6), 598-607.

Wopereis, S., Lefeber, D. J., Morava, E., Wevers, R. A. (2006b) Mechanisms in protein O-glycan biosynthesis and clinical and molecular aspects of protein O-glycan biosynthesis defects: a review. Clin. Chem. 52(4), 574-600.

Wopereis, S., Grünewald, S., Huijben, K. M., Morava, E., Mollicone, R., Van Engelen, B. G., Lefeber, D. J., Wevers, R.A. (2007) Transferrin and apolipoprotein C-III isofocusing are complementary in the diagnosis of $\mathrm{N}$ - and O-glycan biosynthesis defects. Clin. Chem. 53(2), 180-187.

Xia, B., Zhang, W., Li, X., Jiang, R., Harper, T., Liu, R., Cummings, R. D., He, M. (2013) Serum N-glycan and O-glycan analysis by mass spectrometry for diagnosis of congenital disorders of glycosylation. Anal. Biochem. 442(2), 178-185.

Zeevaert, R., De Zegher, F., Sturiale, L., Garozzo, D., Smet, M., Moens, M., Matthijs, G., Jaeken, J. (2013) Bone dysplasia as a key feature in three patients with a novel congenital disorder of glycosylation (CDG) type II due to a deep intronic splice mutation in TMEM165. JIMD Rep. 8, 145-152. 